\title{
Identification of blue and white porcelains from Jiajing official kiln and Dehua Housuo kiln by chemical compositions
}

\author{
Jin Chen, ${ }^{1 *}$ Rongwu Li, ${ }^{2}$ Dawei Yang, ${ }^{3}$ Jiongxin Zhen, ${ }^{4}$ Lifang Chen, ${ }^{4}$ \\ Huansheng Cheng, ${ }^{5}$ Guoxia Li ${ }^{6 *}$ \\ ${ }^{1}$ College of Biochemical Engineering, Beijing Union University, Beijing, 100023, P. R. China \\ ${ }^{2}$ Department of Physics, Beijing Normal University, Beijing, 100875, P. R. China \\ ${ }^{3}$ Henan Broadcast Television University, Zhengzhou, 450003, P. R. China \\ ${ }^{4}$ Dehua Ceramics Museum, Dehua, 362500, P. R. China \\ ${ }^{5}$ Institute of Modern Physics, Fudan University, Shanghai, 200433, P. R. China \\ ${ }^{6}$ Department of Physics, Zhengzhou University, Zhengzhou, 450001, P. R. China
}

Keywords: Blue and White porcelains, Jiajing official kiln, Dehua Housuo kiln, chemical composition, the raw material source, PIXE, fuzzy cluster analysis

Abstract. A scientific method by combination of PIXE measurement and fuzzy cluster analysis was established to distinguish blue and white porcelains from two different kilns. Twenty two blue and white porcelain samples from Jiajing official kiln and Dehua Housuo kiln are applied for this process . The chemical compositions of glazes or body raw material of these samples were measured by Proton Induced X-Ray Emission (PIXE). The obtained chemical ingredients data were analyzed through fuzzy clustering method. The results indicated that the raw material source of blue and white porcelain bodies from Jiajing official kiln is comparatively more concentrated. In contrast, that of Dehua Housuo kiln is comparatively more scattered. The glazes and body raw material source of blue and white porcelain from these two kilns are obviously different. This report provides a scientific method to determine which kiln a sample comes from.

\section{Introduction}

Because Jiajing emperor adored Taoism, porcelains of his period in Jingdezhen, Jiangxi province was mostly with Taoistic style decoration such as gossip, cloud crane, hart crane, Samsung, slender and elegant decoration lines, freehand but rough drawing. Blue and white porcelain of his official kiln is using "Huiqing" material that is blue in the purple, and not elegant, but rich that is different from Chenghua or Hongzhi style of the middle Ming Dynasty. Jiajing blue and white porcelains are mainly red and green with pure, gorgeous style and create a generation of colorful ceramic production in Chinese porcelain history. Jiajing period is the third peak in porcelain art history following Chenghua peak in Ming Dynasty. Based on the records, the number of Jingdezhen porcelains in Jiajing period is about 600000, which is the biggest number in the history of ceramics in China. Therefore, Jiajing period has the far-reaching impact on future generations and is very important for Chinese ceramics history. ${ }^{[1,2]}$

The Housuo kiln is located in Houston village, Dehua County, Fujian province where five ancient kilns sites have been found as conservation units at the county level including Dacaopu kiln site belong to the Song, Yuan, Ming Dynasty, and other sites belong to Ming Dynasty to the Republic of China period. Since Zhaozong He and Zhaochun He in Ming Dynasty made China White (ivory white) porcelain as rare treasures in Housuo klin, Housuo kiln have become famous for a long time. An

I The National Natural Science Foundation of China (Grant No.: 51172212)

I Corresponding author: Jin Chen, female, born in 1977, a lecture in College of Biochemical Engineering, Beijing Union University. E-mail: jessica0726@163.com

I Corresponding author: Guoxia Li, female, born in 1952, a professor in Department of Physics, Zhengzhou University. E-mail: liguoxia@zzu.edu.cn 
ancient porcelain kiln of late Qing Dynasty has been found on November 30th, 2008 around where is scattered a large number of Qing Dynasty blue and white porcelain layer. After careful checking, the archaeologists discovered that the kiln is at the fall of the Ming Dynasty class kiln, but had collapsed, leaving only ruins. ${ }^{[3]}$

Domestic and foreign scholars have already done a lot of nice work about the blue and white porcelain raw material source, micro structure and coloring. ${ }^{[4-5]}$ However, the blue and white porcelain raw material origin and identification of Jingdezhen Jiajing kiln and Dehua kiln remains to be further studied. The porcelain chemical composition of Jingdezhen Jiajing kiln and Dehua Housuo kiln is the same? What is the relationship between two kilns? How to correctly identify them? There are so many questions that puzzle people. The change of elemental composition in ancient ceramics lies in the different formula of fetal and glaze or the various source of the raw material. Since ancient ceramics usually adopted local materials, the source of their raw material varies in different regions and different periods, which make it possible to identify the source of ancient ceramics based on elemental composition. This paper willreport nondestructive determination of the blue and white porcelain chemical composition by using PIXE technology ${ }^{[6]}$ and studies on chemical composition similarity of porcelain bodies, transparent glaze and blue flower glaze (transparent glaze and blue flower) of two kilns $^{[7]}$ using fuzzy cluster analysis. It provides a scientific method to find out the relationship of their raw material origin between two kilns.

\section{Experimental}

\section{Sample selection}

Ten blue and white porcelain samples from Housuo Kiln were produced in Qing Dynasty, Dehua Fujian area. Another twelve blue and white porcelain samples from Jiajing official Kiln were produced in Jiajing period of Ming Dynasty, Jingdezhen area. The code numbers, production time, kiln names of these representative samples are shown in Table 1.

Table 1. Sample information of Jingdezhen Jiajing and Dehua blue and white porcelain

\begin{tabular}{lllll}
\hline Code No. & Dynasty & Production area & \multicolumn{2}{c}{ Name of kiln } \\
\hline QH01-QH10 & Qing & Dehua, Fujian & Housuo & \\
QHJDZ01-QHJDZ12 & Jiajing, Ming & Jingdezhen, Jiangxi & Official \\
\hline
\end{tabular}

\section{PIXE measurements}

PIXE experiment was done by using NEC9SDH-2 accelerator.[8] The types and contents of chemical components were determined by using external beam PIXE technology. Proton beam with initial energy of $3.0 \mathrm{MeV}$ pass through $7.5 \mathrm{um}$ Kapton film and $10 \mathrm{~mm}$ air layer to the sample. The energy of proton beam is $2.8 \mathrm{MeV}$ when it reaches the sample. The X-ray from the excited sample by proton beam bombardment is measured by ORTEC Si (Li) detector. The energy resolution (FWHM) for $5.9 \mathrm{KeV} \mathrm{X}$-ray on measurement system is $165 \mathrm{eV}$. The chemical composition $(\mathrm{Z}>11)$ of the sample can be determined from the obtained $\mathrm{X}$-rays energy spectrum which was calculated by using GUPIX-96 program. The contents of $\mathrm{Na}_{2} \mathrm{O}, \mathrm{MgO}, \mathrm{Al}_{2} \mathrm{O}_{3}, \mathrm{SiO}_{2}, \mathrm{P}_{2} \mathrm{O}_{5}, \mathrm{~K} 2 \mathrm{O}, \mathrm{CaO}, \mathrm{TiO}_{2}, \mathrm{MnO}, \mathrm{Fe}_{2} \mathrm{O}_{3}$ were measured for body and transparent glaze of each porcelain sample. The contents of $\mathrm{Na}_{2} \mathrm{O}, \mathrm{MgO}$ glaze, $\mathrm{Al}_{2} \mathrm{O}_{3}, \mathrm{SiO}_{2}, \mathrm{P}_{2} \mathrm{O}_{5}, \mathrm{~K}_{2} \mathrm{O}, \mathrm{CaO}, \mathrm{TiO}_{2}, \mathrm{MnO}, \mathrm{Fe}_{2} \mathrm{O}_{3}, \mathrm{CoO}$ were measured for glaze of each porcelain sample. The error of measurement data is about $5 \%$. The results of sample test were shown in Table 2. 
Table 2. Chemical composition of some samples

\begin{tabular}{|c|c|c|c|c|c|c|c|c|c|c|}
\hline Code No. & $\mathrm{Na}_{2} \mathrm{O}$ & $\mathrm{MgO}$ & $\mathrm{Al}_{2} \mathrm{O}_{3}$ & $\mathrm{SiO}_{2}$ & $\mathrm{P}_{2} \mathrm{O}_{5}$ & $\mathrm{~K}_{2} \mathrm{O}$ & $\mathrm{CaO}$ & $\mathrm{TiO}_{2}$ & $\mathrm{MnO}$ & $\mathrm{Fe}_{2} \mathrm{O}_{3}$ \\
\hline QH01-b & 0.00 & 0.53 & 18.09 & 74.81 & 0.59 & 4.77 & 0.13 & 0.05 & 0.08 & 0.91 \\
\hline QH02-b & 0.40 & 0.78 & 17.66 & 74.47 & 0.47 & 5.08 & 0.25 & 0.10 & 0.04 & 0.75 \\
\hline QH03-b & 0.50 & 0.68 & 17.03 & 76.33 & 0.39 & 3.77 & 0.10 & 0.06 & 0.09 & 1.02 \\
\hline QH03-wg & 0.40 & 0.39 & 11.92 & 73.21 & 0.48 & 4.45 & 8.06 & 0.04 & 0.16 & 0.88 \\
\hline QH04-wg & 0.00 & 0.32 & 12.97 & 72.53 & 0.51 & 4.23 & 8.05 & 0.11 & 0.12 & 1.09 \\
\hline QH05-wg & 0.38 & 0.63 & 10.57 & 75.64 & 0.88 & 4.85 & 6.32 & 0.06 & 0.09 & 0.55 \\
\hline QH01gbl & 0.84 & 0.49 & 10.09 & 74.65 & 0.60 & 4.41 & 6.13 & 0.11 & 1.66 & 0.80 \\
\hline QH02gbl & 0.74 & 0.11 & 9.48 & 74.29 & 0.55 & 4.23 & 7.21 & 0.11 & 2.25 & 0.78 \\
\hline QH03gbl & 0.00 & 0.21 & 11.00 & 73.97 & 0.58 & 3.75 & 7.40 & 0.11 & 1.83 & 0.77 \\
\hline QHJDZ01-b & 1.93 & 0.68 & 19.80 & 72.60 & 0.38 & 3.43 & 0.15 & 0.08 & 0.02 & 0.81 \\
\hline QHJDZ02-b & 1.13 & 0.38 & 19.30 & 74.20 & 0.22 & 3.65 & 0.16 & 0.06 & 0.03 & 0.87 \\
\hline QHJDZ03-b & 1.03 & 0.53 & 19.90 & 73.80 & 0.15 & 3.43 & 0.15 & 0.07 & 0.03 & 0.94 \\
\hline QHJDZ01-wg & 3.10 & 0.14 & 13.00 & 74.50 & 0.35 & 4.77 & 2.91 & 0.03 & 0.04 & 1.15 \\
\hline QHJDZ02-wg & 2.74 & 0.59 & 13.40 & 70.30 & 0.35 & 4.44 & 7.01 & 0.03 & 0.09 & 0.98 \\
\hline QHJDZ03-wg & 2.10 & 0.57 & 13.40 & 71.60 & 0.11 & 5.04 & 5.28 & 0.04 & 0.09 & 1.83 \\
\hline QHJDZ01-gbl & 2.73 & 0.30 & 13.40 & 73.20 & 0.52 & 4.91 & 2.42 & 0.04 & 0.22 & 1.85 \\
\hline QHJDZ02-gbl & 2.57 & 0.36 & 12.50 & 71.40 & 0.43 & 4.74 & 6.03 & 0.05 & 0.17 & 1.27 \\
\hline QHJDZ03-gbl & 1.62 & 0.36 & 12.50 & 73.00 & 0.36 & 5.11 & 4.58 & 0.05 & 0.14 & 2.10 \\
\hline
\end{tabular}

\section{Fuzzy cluster analysis}

Fuzzy clustering analysis (FCA) is a method which can be used for concepts or mathematical models establishment and laws discovery based on fuzzy mathematical theory.[9] The samples were classified into several categories by FCA. Each sample must fall to one and only one category. The raw material origin of each sample can be characterized by its contents of elements or chemical compositions.

\section{Fuzzy clustering analysis of blue and white porcelain bodies}

The dynamic fuzzy clustering analysis of the chemical component contents of blue and white porcelain bodies measured by PIXE technology was shown in Figure 1. From the Chart, you can see the sample classification is related to threshold value $\lambda$. According to the theoretical value, the optimum threshold is set at 0.751 . Then the samples could be divided into two categories as follows. 


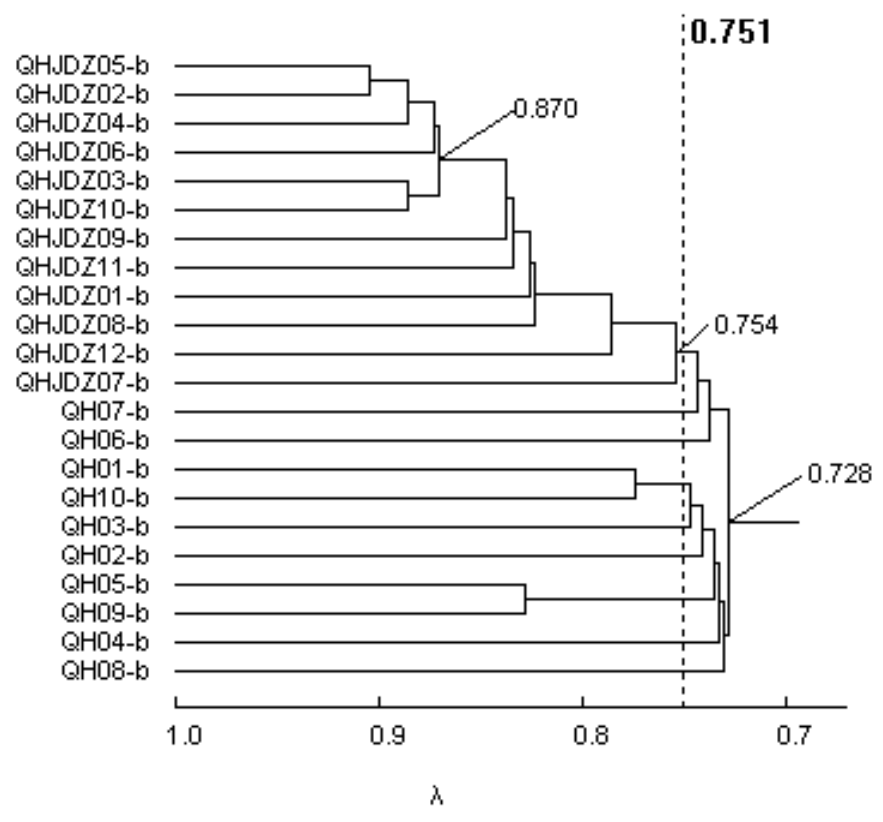

Fig 1. Active FCA chart of blue and white porcelain bodies from Jiajing official and Dehua Housuo kilns

The first category: this category contains 12 samples from QHJDZ05-b to QHJDZ07-b that are from Jiajing official kiln. When $\lambda=0.754$, these samples were classified as one category so the origin of these samples is relatively close. In this category, the relationship of the samples from QHJDZ05-b to QHJDZ10-b is more close because these samples were already as one category even $\lambda=0.870$. It indicates that the raw material origin of these five samples is closer than others.

The second category: This category includes 10 samples from QH07-b to QH08-b that are from Dehua Housuo kiln. When $\lambda=0.728$, these samples are classified as one category. So the body material origin of these samples is relatively close.

\section{Fuzzy clustering analysis of blue and white porcelain transparent glaze}

Figure 2 showed the dynamic fuzzy clustering analysis of chemical component contents of blue and white porcelain transparent glaze such as $\mathrm{Na}_{2} \mathrm{O}, \mathrm{Al}_{2} \mathrm{O}_{3}, \mathrm{SiO}_{2}, \mathrm{P}_{2} \mathrm{O}_{5}, \mathrm{~K}_{2} \mathrm{O}, \mathrm{Fe}_{2} \mathrm{O}_{3}$. When $\lambda=0.770$, the samples can be divided into the following two categories:

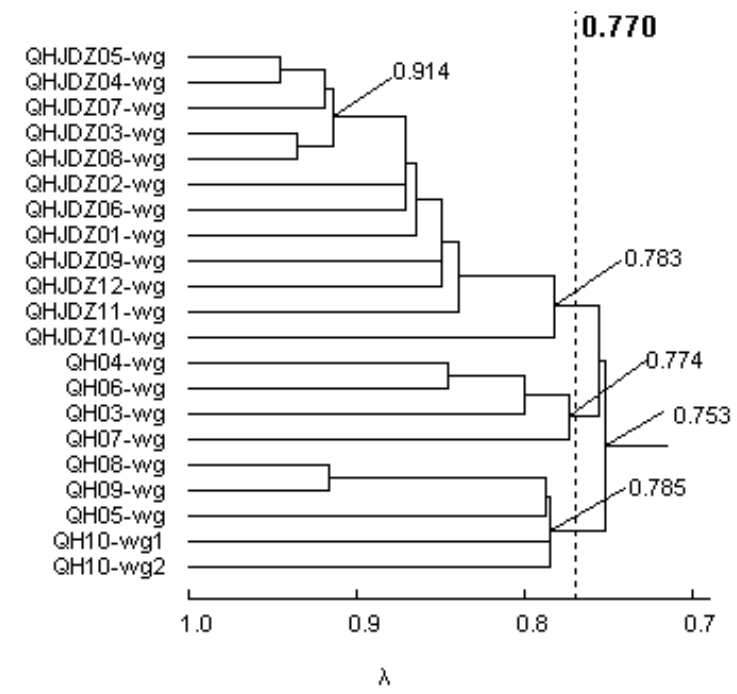

Fig 2. Dynamic FCA chart of blue and white porcelain transparent glaze samples from Jiajing official and Dehua Housuo kilns. 
The first category: this category includes 12 samples from QHJDZ05-wg to QHJDZ10-wg which are from Jiajing official kiln. When $\lambda=0.783$, these samples were classified as one category so the origin of these samples is relatively close. In this category, the relationship of the samples from QHJDZ05-wg to QHJDZ08-wg is more close because these samples were already as one category even $\lambda=0.914$. It indicates that the raw material origin of these five samples is closer than others.

The second category: this category includes 8 samples from QH04-wg to QH10-wg2 which are from Dehua Housuo kiln. When $\lambda=0.753$, these samples are classified as one category. This category can be divided into two parts: the first part includes 4 samples from QH04-wg to QH07-wg. When $\lambda=$ 0.774, these samples get into one class. The second part includes another 4 samples from QH08-wg to QH10-wg2 which got into one class at $\lambda=0.785$. The results of chemical composition measurement are almost same from two different parts of porcelain transparent glaze sample QH10-wg.

\section{Fuzzy clustering analysis of blue and white glaze}

The dynamic FCA chart of blue and white glaze was shown in Figure 3 . When $\lambda=0.701$, the blue and white porcelain samples can be divided into the following two categories:

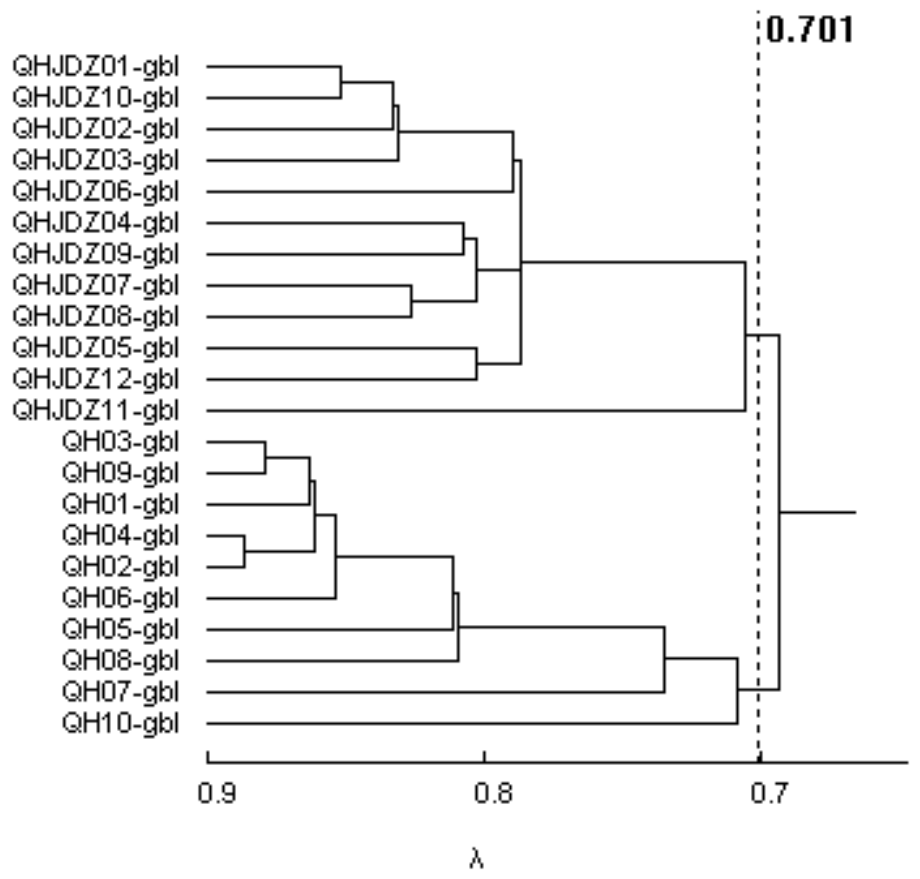

Fig 3. The Jiajing kiln and Dehua kiln porcelain glaze by fuzzy cluster analysis chart

The first category: this category includes 12 samples from QHJDZ01-gbl to QHJDZ11-gbl that were produced at Jiajing official kiln. When $\lambda=0.787$, the 11 samples from QHJDZ01-gbl to QHJDZ12-gbl were already classified as one category, the glaze sources and formula of these samples are closer. All of the 12 samples from Jiajing klin clustered into one category at $\lambda=0.706$, glaze source and formula of these samples is relatively close.

The second category: So the body material origin of these samples is relatively close. From QH03-gbl to QH10-gbl, 10 samples are in another category that is from Dehua Housuo kiln. Eight samples got into one category at $\lambda=0.709$. In this subclass, 6 samples from QH03-gbl to QH06-gbl are closer because they already in the same category at $\lambda=0.853$.

\section{Analysis and discussion}

Based on Table 2, we can see that the chemical composition of blue and white porcelains from Jiajing official and Dehua Huosuo kilns is different. The $\mathrm{Na}_{2} \mathrm{O}$ content of the blue and white porcelain bodies from Jiajing official kiln was higher than that of Dehua Housuo kiln. The $\mathrm{Al}_{2} \mathrm{O}_{3}$ content in most 
Jiajing official klin's blue and white porcelain bodies was higher than that of Dehua Housuo kiln. However, the $\mathrm{K}_{2} \mathrm{O}$ content of the blue and white porcelain bodies from Jiajing official kiln was lower than that of Dehua Housuo kiln. The $\mathrm{Na}_{2} \mathrm{O}$ contents of Jiajing official klin's blue and white porcelain transparent glaze was significantly higher than that of Dehua huosuo kiln's. And the $\mathrm{Al}_{2} \mathrm{O}_{3}$ content in most Jiajing official klin's blue and white porcelain transparent glazes was higher than that of Dehua Housuo kiln. The content of $\mathrm{Na}_{2} \mathrm{O}$ in blue and white glazes of Jiajing official klin was higher than that of Dehua Housuo kiln. The $\mathrm{Al}_{2} \mathrm{O}_{3}, \mathrm{Fe}_{2} \mathrm{O}_{3}$ contents in most Jiajing official klin's blue and white glaze was higher than that of Dehua Housuo kiln, while $\mathrm{SiO}_{2}$ content of Jiajing official klin's blue and white glaze was lower than that of Dehua huosuo kiln.

Figure 1 showed the blue and white porcelain body sample in different classes which indicates that their body material sources are different. From Figure 2, we can see two kilns' blue and white porcelain transparent glaze samples are also divided in different classes, indicating that their transparent glaze sources are also different. Figure 3 showed two kilns' blue and white glaze samples were divided into different classes, suggesting that their blue and white glaze sources or recipe are significantly different.

Due to traffic inconvenience, the raw material of ancient ceramics was always obtained from klin nearby. The chemical component differences of porcelain bodies, transparent glaze, blue and white glaze in two kilns is due to the difference of raw material source and formula of glaze. ${ }^{[10]}$

\section{Conclusions}

Based on the above studies on the samples, the following conclusions can be drawn:

[1] The raw material source of blue and white porcelain bodies produced in Jiajing official kiln was comparatively concentrated. In contrast, the raw material source of Dehua Housuo kiln was more scattered. The raw material sources of porcelain bodies of these two kilns are different.

[2] The raw material sources of blue and white porcelain transparent glaze produced in these two kilns are different.

[3] The material source and recipe of blue and white glaze of these two kilns is significantly different.

[4] It can be clearly identified which klin the blue and white porcelain sample was from by PIXE measurement and fuzzy clustering analysis.

\section{Acknowledgements}

This work was financially supported by the National Natural Science Foundation of China (Grant No.: 51172212). We thank Xinyuan Liu from Jingdezhen ceramic archaeological Institute, Yunpeng Huang from the Jingdezhen international ceramic culture exchange center and staffs from Dehua kiln museum for sample providing. We also thank Prof. Huansheng Cheng from Modern Physics Research Institute of Fudan University for PIXE measurement.

\section{References}

[1] X. Feng, Chinese Ceramics (Revised Edition), Shanghai: Shanghai Classics Publishing House (2001) 518-525.

[2] S. Fu, Y. Sun, Origin and development of Dehua blue and white porcelain, Dehua Ceramics Research Conference Proceeding (2002) 207-211.

[3] Baidu Encyclopedia, Dehua Housuo kiln entries, www.baike.baidu.com/view/6687122.htm.

[4] Y. Chen, Y. Guo, Z. Zhang, Chinese blue-and-white porcelain and blue pigment research, Journal of the Chinese Ceramic Society 6 (4) (1978) 225-241.

[5] J. Wu, J. Li, Z. Deng, etc., Jingdezhen ancient Chinese porcelains dynastic research, China Science series E 34 (2004) 516 524. 
[6] R. Li, W. Zhao, W. Zhao, etc., Three representative glaze colors of Ru and Jun Guan porcelain raw materials PIXE analysis, Journal of Beijing Normal University (Natural science edition) 42 (2) (2006) $144 \sim 149$.

[7] G. Li, H. Sun, R. Li, etc., Three Jun Official porcelain glaze and modern top-grade Jun by neutron activation analysis, Journal of the Chinese Ceramic Society 36 (6) (2008) 816-820.

[8] H. Cheng, D. Zhu, Y. Huang, PIXE studies of Jiajing porcelains, editors H. Luo, X. Zheng. 'the 09 ancient ceramic science and technology 7 International Symposium (ISAC' 09). Shanghai: Shanghai Science and Technology Literature Press (2009) 392 398.

[9] H. Chen. The application of fuzzy mathematics in national economy, Wuhan: Huazhong University of Science \& Technology Press (1994) 97-148.

[10] R. W. Li, G. Chen, D. w. Yang, J. X. Zheng, L. F. Chen, H. S. Cheng, The raw material sources and nondestructive indentification study of the blue and white porcelain made in Jiajing official kiln and Dehua Housuo kiln. Advanced Materials Research, volume 815, 833, Progree in Materials Science and Engineering: ICMSE (2013). 